\title{
CXCL13 in patients with facial palsy caused by varicella zoster virus and Borrelia burgdorferi: a comparative study
}

\author{
Johan Lindström $^{\text {a,b,*, Daniel Bremell }}{ }^{\mathrm{a}, \mathrm{b}}$, Anna Grahn ${ }^{\mathrm{a}, \mathrm{b}}$, Kaj Blennow ${ }^{\mathrm{c}, \mathrm{d}}$, \\ Henrik Zetterberg ${ }^{\mathrm{c}, \mathrm{d}, \mathrm{e}, \mathrm{f}}$, Marie Studahl ${ }^{\mathrm{a}, \mathrm{b}}$ \\ a Department of Infectious Diseases, Sahlgrenska University Hospital, Gothenburg, Sweden \\ b Department of Infectious Diseases, Institute of Biomedicine, Sahlgrenska Academy, University of Gothenburg, Sweden \\ c Department of Psychiatry and Neurochemistry, Institute of Neuroscience and Physiology, Sahlgrenska Academy, University of Gothenburg, Sweden \\ d Clinical Neurochemistry Laboratory, Sahlgrenska University Hospital, Mölndal, Sweden \\ e Department of Neurodegenerative Disease, UCL Institute of Neurology, Queen Square, London, UK \\ ${ }^{\mathrm{f}}$ UK Dementia Research Institute at UCL, London, UK
}

\section{A R T I C L E I N F O}

\section{Article history:}

Received 27 January 2020

Received in revised form 11 May 2020

Accepted 23 May 2020

Available online $\mathrm{xxxx}$

\section{Keywords:}

CXCL13

Cerebrospinal fluid

Lyme neuroborreliosis

Facial paralysis

Varicella zoster virus

\begin{abstract}
A B S T R A C T
High cerebrospinal fluid (CSF) concentrations of the chemokine CXCL13 have been associated with Lyme neuroborreliosis (LNB), and have recently been studied as a potential diagnostic marker. It has proven difficult to establish a reliable diagnostic cut-off, possibly in part due to heterogenicity of case-control groups. Our purpose was to investigate CSF CXCL13 concentrations in patients with similar clinical presentations, facial palsy. We retrospectively included patients with facial palsy associated with LNB $(n=21)$, or varicella zoster virus $(\mathrm{VZV})(n=26)$. Median CXCL13 concentrations were significantly higher in patients with LNB facial palsy compared to VZV facial palsy. Receiver-operating characteristic analyses yielded an optimal cut-off concentration at $34.5 \mathrm{pg} / \mathrm{mL}$ (sensitivity $85.7 \%$, specificity of $84.6 \%$ ), lower than that in previous studies. Although the analysis has potential, it is still not adequately established that CXCL13 provides additional, clinically useful, diagnostic infor-
\end{abstract} mation over current recommendations.

(C) 2020 The Authors. Published by Elsevier Inc. This is an open access article under the CC BY license (http:// creativecommons.org/licenses/by/4.0/).

\section{Background}

Lyme neuroborreliosis (LNB) is a central nervous system (CNS) infection caused by the arthropod-borne spirochete Borrelia burgdorferi $\mathrm{Bb})$. According to European guidelines, a definitive diagnosis of LNB requires a combination of typical clinical symptoms, pleocytosis in the cerebrospinal fluid (CSF) and positive $B b$ antibody index as evidence of intrathecal antibody production (Mygland et al., 2010). Unfortunately, there are several limitations to this definition. Antibody production can be undetectable up to 6 weeks from emergence of symptoms, and can persist for several years after initial disease (Hansen and Lebech, 1992; Hammers-Berggren et al., 1993). Consequently, diagnosis of both early LNB and suspected reinfection is problematic, as the presence of lymphocytes in the CSF is non-specific to LNB.

In an effort to improve diagnostics in CNS infections, high concentrations of the chemokine CXCL13 in CSF has emerged as a possible marker of LNB (Rupprecht et al., 2005). CXCL13, also known as B lymphocyte chemoattractant, is likely important as part of the immune response

\footnotetext{
* Corresponding author. Tel.: +46313439316; fax: +4631847813.

E-mail address: johan.lindstrom@gu.se (J. Lindström).
}

in LNB (Rupprecht et al., 2009). CXCL13 is produced in the CNS in a rapid response to infection, and is detectable before antibodies are present (Senel et al., 2010).

An increasing number of studies have reliably detected significantly higher concentrations of CXCL13 in CSF from patients with LNB compared to other CNS infections and inflammatory diseases (Rupprecht et al., 2005; Ljostad and Mygland, 2008; Senel et al., 2010; van Burgel et al., 2011; Barstad et al., 2017), and it has also been shown that concentrations decline after treatment (Senel et al., 2010; Schmidt et al., 2011; Bremell et al., 2013). Exceptions where similarly increased concentrations may be seen are cryptococcosis, neurosyphilis, tuberculous meningitis and CNS lymphoma (Rupprecht et al., 2009; Marra et al., 2010; Schmidt et al., 2011; van Burgel et al., 2011; Rubenstein et al., 2013). In some of the studies, analyses have been performed with receiver operating characteristic (ROC) analyses, yielding a variety of suggested cut-off concentrations for CSF CXCL13, ranging from $18 \mathrm{pg} / \mathrm{mL}$ (Barstad et al., 2017) to $1224 \mathrm{pg} / \mathrm{mL}$ (Schmidt et al., 2011). In a recently published meta-analysis by Rupprecht et al. (Rupprecht et al., 2018), including 18 studies, a CSF CXCL13 cut-off at $162 \mathrm{pg} / \mathrm{mL}$ was found optimal, at a pooled sensitivity of $89 \%$ and a specificity of $96 \%$, or if only including cross-sectional studies, $91 \mathrm{pg} / \mathrm{mL}$. 
There is no established reference method for CXCL13, so there may be method-related explanations for the varying cut-off concentrations (Henningsson et al., 2016), although several of the studies use the same commercial kit in their analyses. In addition, the patients included in previous studies have presented with a variety of clinical syndromes and duration of symptoms before sampling, which raises questions on comparability.

Facial palsy is a common manifestation of LNB, but can also be caused by varicella zoster virus (VZV) reactivation, traditionally named Ramsay Hunt syndrome (RHS)(Sweeney and Gilden, 2001). Improved diagnostics, such as detection of VZV DNA by PCR of patient CSF, enables patients to be diagnosed with VZV facial palsy regardless of the presence of blisters associated with classical RHS. In the patient with facial palsy, the clinical presentation including associated symptoms may be non-specific, making it impossible to distinguish VZV facial palsy from LNB facial palsy before the results of etiological diagnostics are available. Additionally, CSF pleocytosis is present in both conditions, and in the case of LNB, antibody production may be absent if the duration of symptoms is short. A comparison of CSF CXCL13 in such similar and relevant patient groups has so far not been performed.

\section{Patients and methods}

\section{Patients}

A total of 21 patients with peripheral facial palsy associated with LNB were included from two patient cohorts previously included in unrelated prospective studies on LNB (Bremell and Hagberg, 2011). All patients were examined at the Department of Infectious Diseases, Sahlgrenska University Hospital, Gothenburg, Sweden, between 2000 and 2013, and had a lumbar puncture performed before the initiation of antibiotic treatment. Diagnostic criteria for LNB were: peripheral facial palsy, mononuclear pleocytosis in CSF ( $>5$ cells $/ \mu \mathrm{L})$ and Borreliaspecific intrathecal antibody production in CSF demonstrated with a positive $\mathrm{CSF}$ /serum antibody index (AI), in accordance with European Federation of the Neurological Societies (EFNS) guidelines (Mygland et al., 2010). Patients fulfilling 2 out of 3 criteria are defined as possible LNB according to EFNS guidelines. Since peripheral facial palsy may be present before development of Borrelia-specific antibodies, a history of erythema migrans within 3 months before onset of neurological symptoms was also considered diagnostic in absence of a positive CSF/ serum antibody index.

Twenty-six patients with VZV facial palsy were obtained from a related study on other CSF biomarkers (Lindstrom et al., 2016). These patients were retrospectively identified by detection of VZV DNA in CSF by quantitative in-house PCR, through CSF samples sent to the Virological Laboratory of Sahlgrenska University Hospital during 2002-2013. Subsequently, facial palsy was identified in medical records, and patients with other coexisting CNS diseases were excluded.

As a control group, CSF samples from 52 subjects presenting with headache or psychoneurotic symptoms were used. Controls did not have 1 ) increased lymphocyte counts or protein concentrations in the CSF, 2) concomitant CNS disease or CNS disease identified within 1 year after sampling, 3) increased C-reactive protein concentrations in blood, or 4) pathological findings at neurological examination.

\subsection{CSF samples and analyses}

Two different tests were used to analyze Bb-specific antibodies in CSF and serum during the study period. Until June 26, 2006, samples were analyzed for IgG and IgM with the Dako Lyme Borreliosis ELISA kit using purified native B. burgdorferi flagellum as antigen (Dako Cytomation A/S, Glostrup, Denmark). From June 26, 2006, samples were analyzed with the Liaison chemoluminescence immuno-assay (CLIA), using recombinant VlsE as antigen for IgG and recombinant OspC as antigen for IgM (Diasorin, Saluggia, Italy). Cut-off values for positive samples were according to the manufacturers' instructions.
Until June 26, 2006, the Bb-antibody index was calculated using the optical density (OD) values from the Dako Lyme Borreliosis ELISA test using the formula (OD-CSF/OD-serum) x OD-CSF - OD-serum) where antibody index values $>0.3$ were considered positive (Hansen and Lebech, 1991). From June 26, 2006, Bb-antibody index was calculated as the ratio of the CSF/serum quotient of specific antibodies to the corresponding CSF/serum quotient of total immunoglobulins as described by Reiber and Lange. Antibody index values $>1.4$ were considered positive (Reiber and Lange, 1991).

VZV DNA analysis of CSF samples, with a detection limit of 100 copies/mL, was performed by quantitative in-house PCR previously described (Persson et al., 2009), as part of diagnostic procedure of symptoms.

CXCL13 was measured in stored CSF samples by ELISA (R\&D Systems) according to manufacturer recommendations, with a lower limit of quantification of $7.8 \mathrm{pg} / \mathrm{mL}$. CSF samples from patients with VZV facial palsy had been stored at $-70{ }^{\circ} \mathrm{C}$ or $-20{ }^{\circ} \mathrm{C}$. CSF samples from control subjects and LNB patients had been continually stored at $-70^{\circ} \mathrm{C}$ as part of a biobank where procedures include dividing CSF samples into smaller aliquots to eliminate the need for thawing and refreezing.

\subsection{Statistics}

Statistical analysis was performed using Graphpad Prism 8.4.1 (GraphPad Software, San Diego, USA). CXCL13 values under the detection limit of $7.8 \mathrm{pg} / \mathrm{mL}$ were assigned a value of $3.9 \mathrm{pg} / \mathrm{mL}$ for graphical purposes. Mann-Whitney $U$ test was used for comparison between the 2 groups with LNB facial palsy and VZV facial palsy, Kruskal-Wallis test for comparisons including the control group. Receiver operating characteristic (ROC) curve analysis was performed, using the Youden index (sensitivity + specificity-1) to define optimal cut-offs. Numbers are presented as median and range unless otherwise is indicated.

\subsection{Ethics}

The Regional Ethical Review Board at the University of Gothenburg approved the study (Dnr 385-95 and 664-13).

\section{Results}

\subsection{Patient and control characteristics}

Patient and CSF characteristics are shown in Table 1. Out of the 21 patients with LNB and peripheral facial palsy, all but one patient fulfilled EFNS criteria for definitive LNB. One patient, who had not developed specific antibodies, had a recent history of erythema migrans.

Table 1

Clinical data and CSF findings of the included patients with LNB facial palsy, VZV facial palsy, and controls.

\begin{tabular}{|c|c|c|c|c|}
\hline & $\begin{array}{l}\text { LNB } \\
(n=21)\end{array}$ & $\begin{array}{l}\text { VZV } \\
(n=26)\end{array}$ & $\begin{array}{l}\text { Controls } \\
(n=52)\end{array}$ & $P$ value \\
\hline Sex M/F & $10 / 11$ & $14 / 12$ & $22 / 30$ & \\
\hline Age (years) & $55(7-74)$ & $60(17-93)$ & $60(16-87)$ & 0.64 \\
\hline $\begin{array}{l}\text { Symptom duration } \\
\text { (days) }\end{array}$ & $4(1-35)$ & $2(-9-57)$ & & 0.034 \\
\hline CSF cell count $(\mathrm{no} / \mu \mathrm{L})$ & $153(17-722)$ & $105(3-588)$ & & 0.28 \\
\hline CSF protein (mg/L) & $\begin{array}{l}839 \\
(267-2850)\end{array}$ & $\begin{array}{l}412 \\
(124-1000)\end{array}$ & & 0.0016 \\
\hline $\begin{array}{l}\text { Time in freezer } \\
\text { (years) }\end{array}$ & $6(1-14)$ & $2.5(1-12)$ & & 0.011 \\
\hline CXCL13 (pg/mL) & $\begin{array}{l}2041 \\
(22-36,924)\end{array}$ & $\begin{array}{l}8.5 \\
(<7.8-437)\end{array}$ & $<7.8(<7.8-9)$ & $<0.0001$ \\
\hline
\end{tabular}

$L N B=$ Lyme neuroborreliosis; $V Z V=$ Varicella zoster virus. Comparisons were made using the Mann-Whitney $U$ test when comparing the LNB and VZV groups, Kruskal-Wallis test for comparisons including the control group. 
Sex and age distribution were similar in patients with LNB (10 females and 11 males; median age 55, [range 7-74]), in patients with VZV facial palsy (12 females and 14 males; median age 60 years [7-93]) and in control subjects (30 females and 22 males; median age 60 years [16-87]) $(P=0.64)$.

Median number of days between debut of facial palsy and CSF sampling was 4 [range 1-35] for LNB patients and 2 [range -9 to 10] for VZV patients $(P=0.034)$. There was no significant difference in CSF cell count between LNB patients (median 153/ $\mu \mathrm{L}$ [range 17-722]) and VZV patients $(105 / \mu \mathrm{L}$ [range $3-588])(P=0.28)$, but CSF protein was significantly higher in CSF from LNB patients (839 mg/L [267-2850]) compared to VZV patients (412 mg/L [124-1000]).

Localized pain or headache was frequent in both the LNB group (11/ 21 ) and the VZV facial palsy group (26/26), but neurological symptoms outside of the facial area was more prevalent in the LNB group compared to the VZV group, and included typically radicular pain $(n=11$ / 26). In addition, $14 / 26$ of the patients with VZV facial palsy had blisters, most commonly in the ipsilateral ear region, $16 / 26$ had vertigo and 16/ 26 had affected hearing. Two VZV patients were encephalitic and two patients had mild upper limb paresis but no radicular pain.

\subsection{CSF CXCL13 concentrations}

Median CSF concentrations of CXCL13 for facial palsy caused by LNB were $2041 \mathrm{pg} / \mathrm{mL}$ (range 22-36,924), for VZV facial palsy $8.5 \mathrm{pg} / \mathrm{mL}$ (range $<7.8-437$ ); only in one control sample, CXCL13 concentration exceeded the detection limit with a concentration of $9.0 \mathrm{pg} / \mathrm{mL}$ (Fig. 1).

The differences in CXCL13 concentrations between patients with LNB facial palsy and VZV facial palsy were highly significant $(P<0.0001)$.

ROC analyses were performed on the LNB and VZV facial palsy groups to find an optimal cut-off concentration for CXCL13. A cut-off concentration of $34.5 \mathrm{pg} / \mathrm{mL}$ yielded a sensitivity of $85.7 \%$ and a specificity of $84.6 \%$ (Fig. 2a). Limiting the analysis to patients who had undergone lumbar puncture within the first week after debut of facial palsy (13 LNB, $20 \mathrm{VZV}$ ), a cut-off concentration of $86 \mathrm{pg} / \mathrm{mL}$ yielded a sensitivity of $92.3 \%$ and a specificity of $90 \%$ (Fig. $2 b$ ).

The only LNB patient without a positive antibody index had a markedly increased CXCL13 of $17,446 \mathrm{pg} / \mathrm{mL}$. Five patients fulfilling the criteria for definite LNB had CXCL13 concentrations lower than $50 \mathrm{pg} / \mathrm{mL}$. In four of these cases, CSF had been stored for more than 10 years before analysis, and in three of these cases the duration of facial palsy was 2 weeks or more before sampling was performed. In one patient with a CXCL13 of $35 \mathrm{pg} / \mathrm{mL}$, sampling was performed 4 days after debut of facial palsy, and serological examination was highly positive for

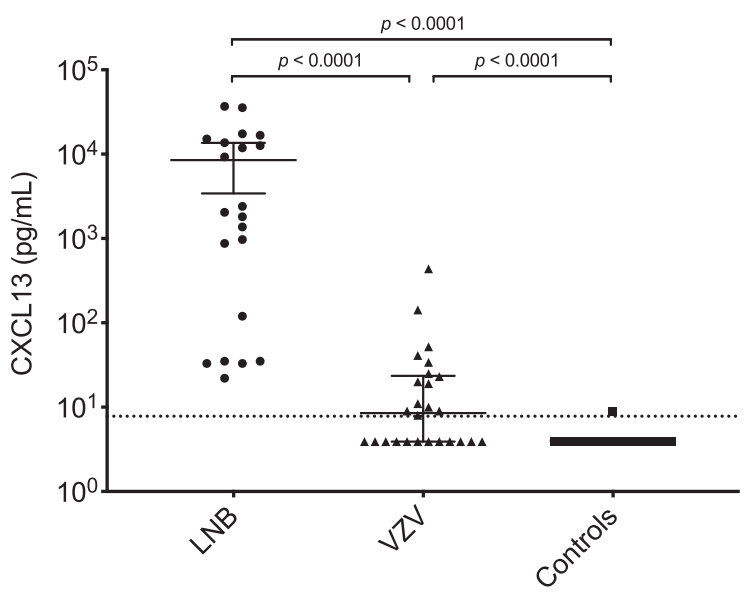

Fig. 1. CXCL13 concentrations in CSF of patients with facial palsy caused by LNB $(n=21)$ compared to VZV $(n=26)$ and controls $(n=52)$ (individual concentrations, median and quartiles). intrathecal Borrelia-specific antibody production. No antibiotic treatment had been given prior to CSF sampling.

Only two patients with VZV facial palsy had CXCL13 increases above $100 \mathrm{pg} / \mathrm{mL}$. First, one 82-year-old female with a CXCL13 of $437 \mathrm{pg} / \mathrm{mL}$ who was admitted with vertigo and after 5 days in-hospital developed facial palsy and blisters typical of zoster oticus. She reported infection with Borrelia 7 years earlier, and Borrelia antibodies were detected in serum but not in the CSF. VZV infection was confirmed through PCR of CSF. Second, one otherwise healthy 29-year-old male who presented in April of 2006, outside of the normal tick-bite season, with typical zoster oticus including associated blisters. Since VZV infection was confirmed through PCR of CSF, no Borrelia-specific analyses were performed. CXCL13 was increased at $142 \mathrm{pg} / \mathrm{mL}$.

\section{Discussion}

In this first comparative study on CXCL13 in patients with facial palsy caused by LNB and VZV, we can confirm significantly higher concentrations of CXCL13 in the CSF of patients with LNB compared to patients with reactivated VZV.

However, the optimal cut-off concentrations for CXCL13 according to the meta-analysis by Rupprecht et al. (Rupprecht et al., 2018) would lead to unacceptably low sensitivity in our material. A cut-off at $162 \mathrm{pg} / \mathrm{mL}$ corresponds to a sensitivity of $71.4 \%$ including all our patients, if we only include patients where sampling was performed in the first week after debut of facial palsy, the sensitivity increases to $84.6 \%$, both with a specificity over $90 \%$. When performing the ROC analyses, our intention was to further show the diversity in CSF CXCL13 expression, and to highlight the difficulties in establishing a reliable cutoff, rather than proposing one of our own. More than half the patients with VZV facial palsy had increased concentrations of CXCL13, weakening specificity of the analysis, but the main finding of low sensitivity for diagnosing LNB using the proposed cut-offs, is related to the relatively low increase of CXCL13 in the CSF from our LNB patients.

There could be multiple explanations to the relatively low increases of CXCL13 shown in the CSF of some of our patients compared to other studies. Selecting patients primarily based on antibody index suggests that enough time has passed since time of infection to allow the immune response to produce antibodies, potentially downstream from early infection where the highest concentrations of CXCL13 are detected (Senel et al., 2010). Isolated facial palsy as a symptom may also have a lesser potential to induce the inflammatory response, being more localized, compared to a more widespread infectious process in the CNS. An additional consideration is time from sampling to analysis in a retrospective study such as ours. There are indications that CXCL13 may degrade over time (Schmidt et al., 2011), and in some cases our samples had been stored for up to 14 years. However, in one study, storage of CSF samples in $-20^{\circ} \mathrm{C}$ for up to 5 years did not result in significantly lower concentrations (van Burgel et al., 2011), and in another study, when the same CSF sample was divided and freeze-thawed up to 5 times, CXCL13 concentrations were unaltered (Hytonen et al., 2014). Although our findings of low increases in CSF CXCL13 are unusual, similar results have been previously described. In Barstad et al. (Barstad et al., 2017) 4/59 children with definite LNB had CSF CXCL13 concentrations $<81 \mathrm{pg} / \mathrm{mL}$, and in Picha et al. (Picha et al., 2016) 3/44 adults with definite LNB had CSF CSXCL13 concentrations $<131 \mathrm{pg} / \mathrm{mL}$. Regardless, the results suggest that methods for measuring this biomarker should be harmonized and, preferably, standardized to a certified reference material.

The results from the present study indicates that analysis of CSF CXCL13 may be of use in the differential workup of patients presenting with facial palsy, although there is still uncertainty regarding optimal cut-offs and applicability. Additionally, studies on CSF CXCL13, including ours, have been performed almost exclusively on European patients, and differences in clinical presentations as well as Borrelia subspecies makes it difficult to draw conclusions regarding CSF CXCL13 in other 

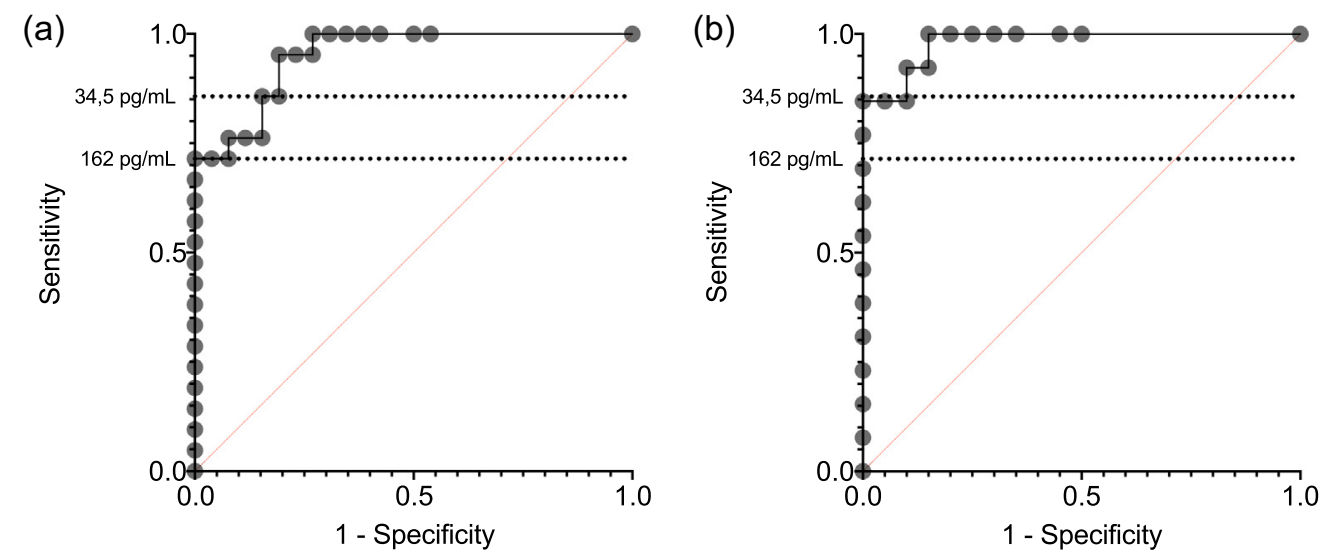

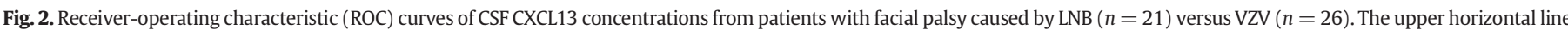

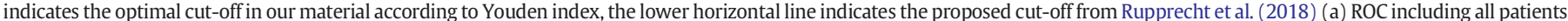
(b) ROC including LNB $(n=13)$ and VZV $(n=20)$ patients sampled within 1 week of debut of symptoms.

geographic settings. The added value of the CXCL13 analysis might be in patients who have not yet developed CSF pleocytosis and/or antibody response to Borrelia due to the short duration of their illness, or in patients with a previous history of LNB, as positive AI may persist for years (Hansen and Lebech, 1992; Hammers-Berggren et al., 1993). In our study, all but one patient had a positive AI only days after debut of facial palsy, implicating that it is a symptom that may develop relatively late in LNB. In addition, according to current EFNS guidelines for possible LNB (Mygland et al., 2010), many patients are prescribed antibiotics when CSF cell count is found to be positive, before the results from the antibody analysis are available. As such, the added benefit of CXCL13 would be to avoid unnecessary antibiotic prescriptions and move focus to potential differential diagnoses. In a newly published study by Pietikäinen et al. (Pietikainen et al., 2018), the authors advocate for a new CXCL13 lateral flow assay, suitable for point-of-care testing. Such an assay could potentially widen the indication for CXCL13 testing, and have an impact on clinical practice.

Even if CSF CXCL13 has potential as a useful analysis in the clinical setting, further studies on CNS infections with similar clinical presentations are warranted, primarily focusing on patients only fulfilling the criteria of possible LNB, where the potential added diagnostic value is greatest.

\section{Acknowledgements}

The study was financed by grants from the Swedish state under the agreement between the Swedish government and the country councils, the ALF-agreement (\#ALFGBG-73490; \#ALFGBG-74050; \#ALFGBG720931; \#ALFGBG-715986). HZ is a Wallenberg Academy Fellow supported by grants from the Swedish Research Council (\#2018-02532), the European Research Council (\#681712). KB holds the Torsten Söderberg Professorship in Medicine at the Royal Swedish Academy of Sciences, and is supported by the Swedish Research Council (\#201700915), and the Swedish Alzheimer Foundation (\#AF-742881), Hjärnfonden, Sweden (\#FO2017-0243).

\section{Declaration of competing interest}

$\mathrm{HZ}$ has served at scientific advisory boards for Roche Diagnostics, Samumed, CogRx and Wave, has given lectures in symposia sponsored by Biogen and Alzecure, and is a co-founder of Brain Biomarker Solutions in Gothenburg AB, a GU Ventures-based platform company at the University of Gothenburg (all outside submitted work). KB has served as a consultant or at advisory boards for Biogen, Lilly, Novartis and Roche Diagnostics, and is a co-founder of Brain Biomarker Solutions in Gothenburg $A B$, a GU Venture-based platform company at the
University of Gothenburg, (all outside submitted work). The other authors report no conflicts of interest.

\section{Author Statement}

Johan Lindström: Methodology, Formal analysis, Writing- Original draft preparation, Visualization. Daniel Bremell: Investigation, Methodology, Writing- Reviewing and Editing. Anna Grahn: Investigation, Writing- Reviewing and Editing. Kaj Blennow: Resources, Validation, Writing- Reviewing and Editing. Henrik Zetterberg: Resources, Investigation, Validation, Writing- Reviewing and Editing. Marie Studahl: Conceptualization, Supervision, Writing- Reviewing and Editing.

\section{References}

Barstad B, Tveitnes D, Noraas S, Selvik Ask I, Saeed M, Bosse F, et al. Cerebrospinal fluid Blymphocyte Chemoattractant CXCL13 in the diagnosis of acute Lyme neuroborreliosis in children. Pediatr Infect Dis J 2017;36(12):e286-92. https://doi.org/10.1097/INF. 0000000000001669

Bremell D, Hagberg L. Clinical characteristics and cerebrospinal fluid parameters in patients with peripheral facial palsy caused by Lyme neuroborreliosis compared with facial palsy of unknown origin (Bell's palsy). BMC Infect Dis 2011;11:215. https:// doi.org/10.1186/1471-2334-11-215.

Bremell D, Mattsson N, Edsbagge M, Blennow K, Andreasson U, Wikkelso C, et al. Cerebrospinal fluid CXCL13 in Lyme neuroborreliosis and asymptomatic HIV infection. BMC Neurol 2013;13:2. https://doi.org/10.1186/1471-2377-13-2.

Hammers-Berggren S, Hansen K, Lebech AM, Karlsson M. Borrelia burgdorferi-specific intrathecal antibody production in neuroborreliosis: a follow-up study. Neurology 1993;43(1):169-75. https://doi.org/10.1212/wnl.43.1_part_1.169.

Hansen K, Lebech AM. Lyme neuroborreliosis: a new sensitive diagnostic assay for intrathecal synthesis of Borrelia burgdorferi-specific immunoglobulin G, a, and M. Ann Neurol 1991;30(2):197-205. https://doi.org/10.1002/ana.410300212.

Hansen K, Lebech AM. The clinical and epidemiological profile of Lyme neuroborreliosis in Denmark 1985-1990. A prospective study of 187 patients with Borrelia burgdorfer specific intrathecal antibody production. Brain : a journal of neurology 1992;115(Pt 2):399-423. https://doi.org/10.1093/brain/115.2.399.

Henningsson AJ, Gyllemark P, Lager M, Skogman BH, Tjernberg I. Evaluation of two assays for CXCL13 analysis in cerebrospinal fluid for laboratory diagnosis of Lyme neuroborreliosis. APMIS 2016;124(11):985-90. https://doi.org/10.1111/apm.12596.

Hytonen J, Kortela E, Waris M, Puustinen J, Salo J, Oksi J. CXCL13 and neopterin concentrations in cerebrospinal fluid of patients with Lyme neuroborreliosis and other diseases that cause neuroinflammation. J Neuroinflammation 2014;11:103. https://doi.org/10. 1186/1742-2094-11-103.

Lindstrom J, Grahn A, Zetterberg H, Studahl M. Cerebrospinal fluid viral load and biomarkers of neuronal and glial cells in Ramsay hunt syndrome. Eur J Neurosci 2016; 44(11):2944-9. https://doi.org/10.1111/ejn.13403.

Ljostad U, Mygland A. CSF B-lymphocyte chemoattractant (CXCL13) in the early diagnosis of acute Lyme neuroborreliosis. J Neurol 2008;255(5):782. https://doi.org/10.1007/ s00415-008-0974-8.

Marra CM, Tantalo LC, Sahi SK, Maxwell CL, Lukehart SA. CXCL13 as a cerebrospinal fluid marker for neurosyphilis in HIV-infected patients with syphilis. Sex Transm Dis 2010; 37(5):283-7. https://doi.org/10.1097/OLQ.0b013e3181d877a1.

Mygland A, Ljostad U, Fingerle V, Rupprecht T, Schmutzhard E, Steiner I, et al. EFNS guidelines on the diagnosis and management of European Lyme neuroborreliosis. Eur Neurol 2010;17(1):8-16. e11-14 https://doi.org/10.1111/j.1468-1331.2009.02862.x. 
Persson A, Bergstrom T, Lindh M, Namvar L, Studahl M. Varicella-zoster virus CNS disease - viral load, clinical manifestations and sequels. J Clin Virol 2009;46(3):249-53. https://doi.org/10.1016/j.jcv.2009.07.014.

Picha D, Moravcova L, Smiskova D. Prospective study on the chemokine CXCL13 in neuroborreliosis and other aseptic neuroinfections. J Neurol Sci 2016;368:214-20. https://doi.org/10.1016/j.jns.2016.05.059.

Pietikainen A, Oksi J, Hytonen J. Point-of-care testing for CXCL13 in Lyme neuroborreliosis Diagn Microbiol Infect Dis 2018;91(3):226-8. https://doi.org/10.1016/j.diagmicrobio. 2018.02.013.

Reiber H, Lange P. Quantification of virus-specific antibodies in cerebrospinal fluid and serum: sensitive and specific detection of antibody synthesis in brain. Clin Chem 1991;37(7):1153-60.

Rubenstein JL, Wong VS, Kadoch C, Gao HX, Barajas R, Chen L, et al. CXCL13 plus interleukin 10 is highly specific for the diagnosis of CNS lymphoma. Blood 2013;121(23): 4740-8. https://doi.org/10.1182/blood-2013-01-476333.

Rupprecht TA, Pfister HW, Angele B, Kastenbauer S, Wilske B, Koedel U. The chemokine CXCL13 (BLC): a putative diagnostic marker for neuroborreliosis. Neurology 2005; 65(3):448-50. https://doi.org/10.1212/01.wnl.0000171349.06645.79.

Rupprecht TA, Plate A, Adam M, Wick M, Kastenbauer S, Schmidt C, et al. The chemokine CXCL13 is a key regulator of B cell recruitment to the cerebrospinal fluid in acute
Lyme neuroborreliosis. J Neuroinflammation 2009;6:42. https://doi.org/10.1186/ 1742-2094-6-42.

Rupprecht TA, Manz KM, Fingerle V, Lechner C, Klein M, Pfirrmann M, et al. Diagnostic value of cerebrospinal fluid CXCL13 for acute Lyme neuroborreliosis. A systematic review and meta-analysis. Clin Microbiol Infect 2018;24(12):1234-40. https://doi.org/ 10.1016/j.cmi.2018.04.007.

Schmidt C, Plate A, Angele B, Pfister HW, Wick M, Koedel U, et al. A prospective study on the role of CXCL13 in Lyme neuroborreliosis. Neurology 2011;76(12):1051-8. https://doi.org/10.1212/WNL.0b013e318211c39a.

Senel M, Rupprecht TA, Tumani H, Pfister HW, Ludolph AC, Brettschneider J. The chemokine CXCL13 in acute neuroborreliosis. J Neurol Neurosurg Psychiatry 2010;81(8): 929-33. https://doi.org/10.1136/jnnp.2009.195438.

Sweeney CJ, Gilden DH. Ramsay hunt syndrome. J Neurol Neurosurg Psychiatry 2001;71 (2):149-54. https://doi.org/10.1136/jnnp.71.2.149.

van Burgel ND, Bakels F, Kroes AC, van Dam AP. Discriminating Lyme neuroborreliosis from other neuroinflammatory diseases by levels of CXCL13 in cerebrospinal fluid. J Clin Microbiol 2011;49(5):2027-30. https://doi.org/10.1128/JCM.00084-11. 